\title{
Detección mediante pirosecuenciación de la mutación nt23o [del4] del gen $A B C B 1$ canino y determinación de su prevalencia en razas de perros pastores en la provincia de Buenos Aires
}

\author{
Detection by pyrosequencing and prevalence of canine ABCB1 gene \\ mutation nt23o [del4] in sheep dog breeds of Buenos Aires province
}

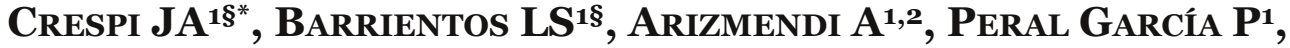 \\ Giovambattista $\mathbf{G}^{1}$ \\ $\S$ Ambos autores contribuyeron de igual manera
}

1. Instituto de Genética Veterinaria Ing. Fernando N. Dulout (IGEVET, UNLP-CONICET LA PLATA). 2. Servicio de Cardiología, Facultad de Ciencias Veterinarias, Universidad Nacional de La Plata, Argentina.

*Correo electrónico del autor de contacto: jcrespi@fcv.unlp.edu.ar

\begin{abstract}
Resumen
El gen $A B C B 1$ codifica para la glicoproteína-P (Pgp), una proteína de membrana que transporta múltiples fármacos fuera de la célula. Este gen se expresa principalmente en la barrera hematoencefálica, cumpliendo también importantes funciones en otros órganos. En caninos, se ha informado una deleción de $4 \mathrm{pb}$ en el exón 4, que genera una terminación prematura y una proteína no funcional. Los animales homocigotos para la mutación presentan neurotoxicidad al administrarles drogas como las avermectinas. Esta mutación ha sido comunicada principalmente en collie, border collie y otras razas de perros pastores. El objetivo del trabajo consistió en desarrollar un método de diagnóstico rápido de la mutación nt23o [del4] del gen $A B C B 1$ basado en pirosecuenciación y validarla en una población local. Se analizaron 72 perros mediante pirosecuenciación y secuenciación directa. Los resultados obtenidos con ambas técnicas evidenciaron $100 \%$ de concordancia. El cálculo de la frecuencia génica del alelo nt230 [del4] (q) mostró diferencias entre las razas tipificadas: collie ( $\mathrm{n}=30$ y q=0,32), border collie $(\mathrm{n}=17 \mathrm{y} \mathrm{q}=0,06)$, pastor de Shetland $(\mathrm{n}=12$ y q=0) y otras $(\mathrm{n}=16$ y q=0). El valor promedio obtenido de frecuencia génica del alelo nt23o [del4] resultó menor al reportado en otros países. En conclusión, el método desarrollado podría ser utilizado para la detección temprana de la mutación nt23o [del4] causal de la deficiencia de Pgp, siendo una herramienta muy útil para la reproducción controlada y la prevención de la neurotoxicidad en los pacientes frente a tratamientos con avermectinas y otras drogas sustrato de la Pgp.
\end{abstract}

\section{Palabras clave}

Perros, glicoproteína P, neurotoxicidad, pirosecuenciación, prevalencia

\begin{abstract}
$A B C B 1$ gene codes for P-glycoprotein (Pgp), a membrane protein that transports multiple drugs out of the cell. It is mainly expressed in the blood-brain barrier, although it also plays a role in other organs. In dogs, a 4 bp deletion in exon 4 of $A B C B 1$ gene has been reported. This mutation results in a premature stop codon and a nonfunctional protein. Homozygous animals for the deletion present neurotoxicity when administered drugs such as avermectins. This mutation is found mainly in collie, border collie and other related dog breeds. The aim of the present work was to develop a pyrosequencing method for rapid diagnosis of the $A B C B 1$ gene nt23o [del4] mutation and validate it by direct sequencing in a local population. A total of 72 dogs were analyzed, obtaining a $100 \%$ agreement between both methods. The genetic variation of the mutated allele (q) showed differences between the evaluated breeds: collie $(\mathrm{n}=$ $30 ; q=0.32)$, border collie $(n=17 ; q=0.06)$, Shetland sheep $\operatorname{dog}(n=12 ; q=0)$, others $(n=13 ; q=0)$. On average, the q value was lower than reported in other countries. Pyrosequencing could be used for early diagnosis of Pgp deficiency, being a very useful tool for controlled reproduction in kennels and for the prevention of neurotoxicity in patients treated with avermectins and other Pgp substrate drugs.
\end{abstract}

\section{Key words}

Dogs, P-glycoprotein, neurotoxicity, pyrosequencing, prevalence

Fecha de recepción: 07/12/2017

ANALECTA Vet 2018; 38(1): 2-8

Fecha de aprobación: 24/04/2018

Impresa ISSN 03655 14-8 Electrónica ISSN 1514-2590

doi.org/10.24215/15142590eo19 


\section{Introducción}

La glicoproteína-P 1 (glicoproteína de permeabilidad, Pgp), también conocida como multidrug resistance protein 1 (Mdr1), protege a los tejidos de la acumulación de xenobióticos y tiene un gran impacto en la distribución de varios fármacos. El amplio espectro de los sustratos de la Pgp incluye antiparasitarios, antibióticos, quimioterápicos, analgésicos, glucocorticoides y antiepilépticos (Fromm, 2003; Löscher \& Potschka, 2005).

La influencia de la Pgp en la disposición de ciertos fármacos se ha demostrado en perros de raza collie y en otras razas de perros pastores (Mealey et al., 2001; Nelson et al., 2003; Roulet et al., 2003). Se ha observado intoxicación grave en respuesta al tratamiento con el fármaco antiparasitario ivermectina y otras avermectinas en una subpoblación de estas razas, lo que demuestra la predisposición genética a una mayor permeabilidad de la barrera hematoencefálica a ciertos fármacos (Schinkel, 1999). Esto permite que la ivermectina ingrese en las neuronas y ejerza efectos sobre los canales de cloruro regulados por ácido $\gamma$-aminobutírico (GABA). Además, otras drogas de uso frecuente en medicina veterinaria, como la doxorrubicina y la vincristina, son sustrato de la Pgp (Stiedl et al., 2017). Mealey et al. (2001), identificaron en una subpoblación de perros de raza collie una deleción de 4 pares de bases (pb) en el exón 4 en el gen $A B C B 1$ (ATPbinding cassette, sub-family $B \quad-M d r / T a p-$, member 1) que se ubica en el cromosoma 14 y que codifica para la proteína Pgp. Posteriormente, esta mutación, denominada nt23o [del4], también fue detectada en perros de la raza pastor australiano sensibles a ivermectina (Nelson et al., 2003). La deleción provoca un cambio de marco de lectura y un codón de detención prematuro con la consecuente Pgp truncada (Mealey et al., 2001). Neff et al. (2004), detectaron el alelo mutante en otras seis razas, incluyendo el pastor inglés, el whippet de pelo largo, el pastor de $\mathrm{McNab}$, el pastor de Shetland y el silken windhound. En estas razas, los veterinarios deben ser cautelosos con el uso de medicamentos sustratos de Pgp. Sin embargo, el tratamiento con dichos fármacos puede ser preferido o incluso necesario en algunos casos clínicos, razón por la cual el análisis genético es de valor para revelar el genotipo $A B C B 1$ ( $m d r 1$ ) del paciente y adaptar la elección del fármaco a cada paciente. Los sustratos de Pgp podrían utilizarse en perros con alelos del gen $A B C B 1$ de tipo normal y evitarse en perros portadores de la deleción nt230 [del4].

Hasta el momento, se han utilizado varios métodos para la detección de esta mutación, como AS-PCR (reacción en cadena de la polimerasa, alelo-específica) (Baars et al. 2007), electroforesis en geles de poliacrilamida de alta resolución (Greyer et al., 2005, Kawabata et al., 2005) y secuenciación directa (Gagliardi et al., 2015).
El objetivo del presente trabajo consistió en desarrollar un método de detección de la mutación nt23o [del4] del gen $A B C B 1$ mediante pirosecuenciación para identificar el genotipo de riesgo de manera más rápida, sencilla y eficiente. De esta manera se pudo determinar su frecuencia en la población de perros pastores de algunas razas en la provincia de Buenos Aires.

\section{Materiales y métodos}

El presente estudio fue aprobado por el Comité Institucional para el Cuidado y Uso de Animales de Laboratorio (CICUAL) de la Facultad de Ciencias Veterinarias de la Universidad Nacional de La Plata, Argentina. Los propietarios de los perros firma-ron un consentimiento informado previo al muestreo.

Se colectaron 72 muestras de sangre entera con anticoagulante EDTA al $6 \%$, por venopunción cefálica o safena. Los animales muestreados fueron perros de diferentes razas provenientes de distintas zonas de la provincia de Buenos Aires: 59 perros pastores (Grupo 1 - Sección 1, Federación Cinológica Internacional) de los cuales 30 eran de la raza collie, 17 de la raza border collie, 12 de la raza pastor de Shetland; también se incluyeron en el estudio 13 perros seleccionados al azar de razas no incluidas en la categoría de perros pastores y que no presentaban hasta el momento, según la bibliografía, informes de esta mutación (2 Airedale terrier, 1 bulldog francés, 2 caniche toy, 2 dóberman, 2 dogo argentino, 1 fox terrier wire, 2 golden retriever y 1 podenco faraónico. Todos los animales utilizados en este trabajo se encuentran registrados dentro del circuito oficial cinológico y cumplen con la condición de no estar emparentados entre sí.

El ADN genómico se extrajo a partir de las muestras de sangre utilizando el kit de purificación Wizard Genomic DNA purification (Promega Madison, WI) de acuerdo a las recomendaciones del fabricante. Los animales se genotipificaron mediante los métodos de secuenciación directa y pirosecuenciación.

El amplificado por PCR de la secuencia de interés se llevó a cabo mediante cebadores (Fmdr1, R-mdr1-M13 e INT-mdr1) diseñados utilizando el programa Annhyb (http://bioinformatics.org/ annhyb/) y la secuencia de ADN reportada en GenBank NM_001003215.2 (http:/www.ncbi.nlm. nih.gov/genbank/) (Tabla 1, Figura1).

Los componentes de cada reacción fueron: 20 ng de ADN molde, buffer de reacción $1 \mathrm{X}$ (50 $\mathrm{mM} \mathrm{KCl}, 11 \mathrm{mM}$ Tris, $\mathrm{pH}=8,30), \mathrm{MgCl}_{2}$ o,5 mM, potenciador $0,08 \mathrm{X}$, dNTP $100 \mu \mathrm{M}$ de cada uno, cebadores $0,2 \mu \mathrm{M}$ cada uno y Taq polimerasa 0,75 U (T-Holmes DNA polymerase, INBIO Highway, Tandil, Argentina).

Para la genotipificación por secuenciación directa se utilizaron los cebadores específicos 
Figura 1. Cebadores utilizados en este estudio. Subrayado: polimorfismo InDel (inserción-deleción), F-mdr1 (cebador directo), R-mdr1M13 (cebador reverso con la secuencia de M13 en su extremo $5^{\prime}$ ) e INT-mdr1 (cebador interno utilizado en pirosecuenciación).

F-mdr1 y R-mdr1-M13 y el ciclado tuvo una temperatura de hibridación de $58^{\circ} \mathrm{C}$. Los productos de PCR fueron purificados con polietilenglicol (PEG) 8000 y secuenciados en un secuenciador automático Genetic Analyzer 3500 (Applied Biosystemsß) utilizando el kit comercial (BigDye ${ }^{\mathrm{TM}}$ Terminator v3.1 Cycle Sequencing Kit - Applied BiosystemThermo Fisher Scientific). Las secuencias obtenidas se alinearon y analizaron utilizando los programas para análisis de secuencias DNAMAN 4.15, Lynnon BioSoft, Vaudreuil, Quebec, Canadá y Chromas 2.4, Technelysium, South Brisbane, Australia.

\begin{tabular}{cccc}
\hline Cebadores & Secuencia $5^{-}-\mathbf{3}^{-}$ & $\begin{array}{c}\text { Temperatura de } \\
\text { hibridación }\end{array}$ & $\begin{array}{c}\text { Tamaño del } \\
\text { fragmento }(\mathbf{p b})\end{array}$ \\
\hline F- $m d r I$ & CATCCATGGAGCTGCACTCCC & $58^{\circ} \mathrm{C}$ & \\
R- $m d r 1$-M13 & M13-TCAAACCTCTAAGATCAGTGCCACA & $58^{\circ} \mathrm{C}$ & 150 \\
INT- $m d r 1$ & GCTGGTTTTTGGAAACATGAC & $52^{\circ} \mathrm{C}$ & \\
M13-Biot & Biot-AGCGCATAACAATTTCACACAGGT & $52^{\circ} \mathrm{C}$ & \\
\hline
\end{tabular}

Tabla 1. Secuencia de los cebadores utilizados en este estudio.

La genotipificación por pirosecuenciación se llevó a cabo utilizando los cebadores F-mdr1, R-mdr1-M13 y M13-Biot. En cuanto a las condiciones de ciclado, la elongación se dividió en dos etapas: la primera, de 15 ciclos, con una hibridación a $58{ }^{\circ} \mathrm{C}$, en la que se amplifica el fragmento utilizando los cebadores específicos F-mdr1, R-mdr1-M13 y, la segunda, en la que se incorpora el cebador biotinilado M13-Biot con 30 ciclos e hibridación a $52{ }^{\circ} \mathrm{C}$. Los productos de PCR fueron purificados captando los fragmentos biotinilados con perlas de sefarosa cubiertas con estreptavidina (Streptavidin SepharoseTM High Performance, GE Healthcare). Los fragmentos biotinilados se inmovilizaron y utilizaron como molde para realizar la reacción de pirosecuenciación, incorporando el cebador interno INT-mdr1 $(0,5 \mu \mathrm{M})$ usando el kit comercial (Pyro Gold Reagent Kit, Biotage, Uppsala, Suecia) y PyroMark Prep Workstation (QIAGEN, Hilden, Alemania). Las muestras se tipificaron en un pirosecuenciador PSQTM96 System Instrument (QIAGEN) y los resultados se analizaron con el programa PSQ HS96A (QIAGEN).

Los cálculos de las frecuencias génicas y genotípicas, el equilibrio de Hardy-Weinberg
(HWE), la heterocigosidad observada (ho) y esperada (he) y la diferenciación génica entre pares de grupos se realizaron utilizando el software Genepop (http://genepop.curtin.edu.au/) con un valor de significancia igual a 0,05.

\section{Resultados}

En este estudio, la totalidad de los animales se genotipificaron mediante los métodos de secuenciación directa y de pirosecuenciación, obteniéndose un $100 \%$ de concordancia entre los resultados obtenidos con ambos métodos (Figura 2). Las frecuencias génicas y genotípicas para el alelo nt230 [del4] del gen $A B C B 1$ canino se calcularon en el total de la muestra y en cada grupo por separado (Tabla 2). La muestra analizada presentó una he de 0,25 y una ho de 0,15. La estimación de HWE en la población total y dentro de cada uno de los cuatro grupos raciales evidenció que solo en el total de la muestra se observó un desvío significativo con respecto a las proporciones teóricas esperadas para el equilibrio $(\mathrm{p}=0,004)$ (Tabla 2).

\begin{tabular}{cccccccccc}
\hline & $\mathrm{n}$ & $(+)$ & $(-)$ & $(+/+)$ & $(-/-)$ & $(+/-)$ & $\begin{array}{c}\text { HWE } \\
\text { (valor de p) }\end{array}$ & $\mathrm{H}_{\mathrm{e}}$ & $\mathrm{H}_{\circ}$ \\
\hline Población total & 72 & 0,85 & 0,15 & $56(0,78)$ & $5(0,07)$ & $11(0,15)$ & 0,004 & 0,25 & 0,15 \\
collie & 30 & 0,68 & 0,32 & $16(0,53)$ & $5(0,17)$ & $9(0,30)$ & 0,099 & 0,43 & 0,30 \\
border collie & 17 & 0,94 & 0,06 & $15(0,88)$ & 0 & $2(0,12)$ & 1,000 & 0,11 & 0,12 \\
pastor de Shetland & 12 & 1 & 0 & $12(1)$ & 0 & 0 & - & - & - \\
otras & 13 & 1 & 0 & $13(1)$ & 0 & 0 & - & - & - \\
\hline
\end{tabular}

Tabla 2. Frecuencias génicas y genotípicas de la mutación nt230 [del4] del gen $A B C B 1$ canino en la provincia de Buenos Aires. HWE: Equilibrio de Hardy-Weinberg; (+): alelo normal; (-): alelo mutado; $(+/+)$ : genotipo homocigota normal, (+/-): genotipo heterocigota; (-/-): genotipo homocigota mutado; (He) heterocigosidad esperada; (Ho): heterocigosidad observada.

La prueba de diferenciación génica entre pares de grupos evidenció diferencias con valores significativos entre la raza collie y los otros tres grupos (Tabla 3). La mayor diferencia se detectó entre la raza collie y el grupo de otras razas $(\mathrm{p}=0,00044)$, seguida por la detectada entre perros de raza collie y el grupo de pastores de Shetland ( $\mathrm{p}=0,00091) \mathrm{y}$, por último, con el grupo de la raza border collie $(\mathrm{p}=0,00286)$. 
a)
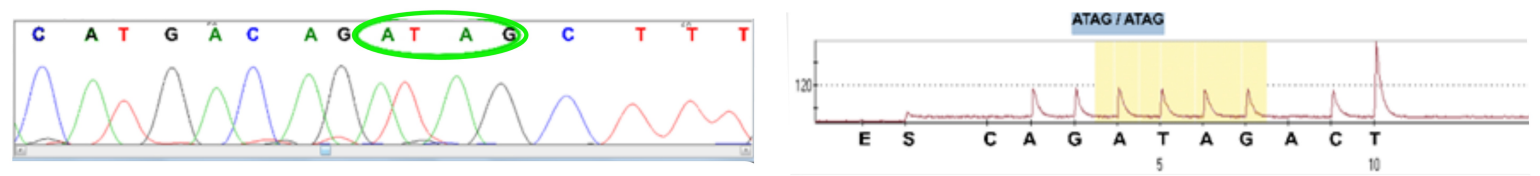

b)
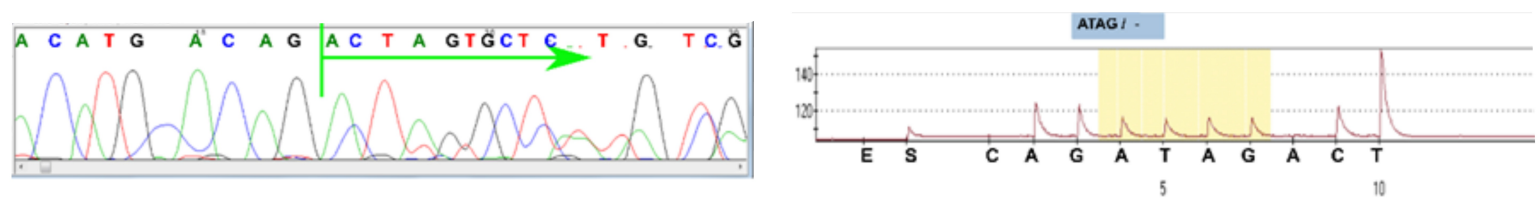

c)

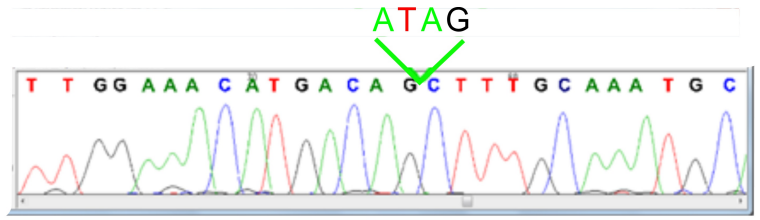

Electroferograma

Serılenciarión diresta

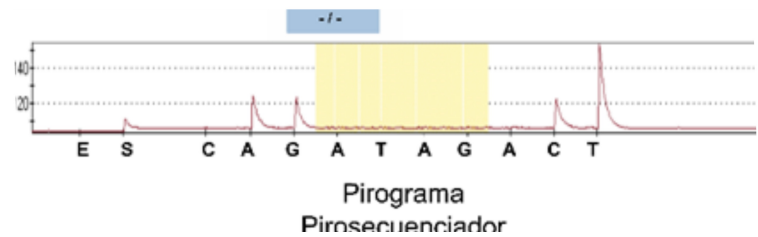

Figura 2. Correlación entre ambos métodos de secuenciación para validar la técnica desarrollada. a) Genotipo homocigota normal (+/ +), b) Genotipo heterocigota (+/-), c) Genotipo homocigota mutado (-/-).

\begin{tabular}{lcc}
\hline Pares de grupos & Valor de p & $\chi^{\mathbf{2}}$ \\
\hline border collie/collie & $0,00286^{*}$ & 11,71 \\
otras/collie & $0,00044^{*}$ & 15,46 \\
otras/border collie & 0,50021 & 1,38 \\
pastor de Shetland/collie & $0,00091^{*}$ & 14,00 \\
pastor de Shetland/border collie & 0,50585 & 1,36 \\
pastor de Shetland/otras & - & - \\
\hline
\end{tabular}

Tabla 3. Diferenciación genética para la mutación nt23o [del4] del gen $A B C B 1$ entre los diferentes grupos de razas utilizados en este estudio (Método de Fisher, valor de significancia $\alpha=$ o,05). $(*)$ Valores significativos.

\section{Discusión}

El método desarrollado de detección de esta mutación resultó ser una alternativa igualmente eficaz con respecto a la secuenciación directa y, a su vez, más eficiente, proporcionando los mismos resultados con un menor costo y un volumen de procesamiento mayor.

Los resultados obtenidos mostraron que la frecuencia del alelo mutado (-) en la población de la raza collie analizada en la región estudiada fue menor $(0,32)$ que la informada en otros países (Tabla 4). En el caso de la raza border collie, la prevalencia del alelo nt23o [del4] en la muestra local fue seis veces mayor que la comunicada por Gramer (2011) $(q=0,01)$ y diez veces mayor que la informada por Greyer et al. (2005) (q = 0,006), Huebner et al. (2007) y Fridova et al. (2016) $(\mathrm{q}=\mathrm{o})$. En cuanto a la población local de la raza Pastor de Shetland, en este estudio no se encontraron animales portadores del alelo nt23o [del4], mientras que en otros países la frecuencia del alelo mutado se encuentra entre el 0,2 (Stiedl et al., 2017) y el o,3 (Huebner et al., 2007; Gramer et al., 2011; Fridova et al., 2016). Estas diferencias pueden ser la consecuencia del efecto del grupo de perros fundador de las poblaciones de cada país o de la deriva génica producto del reducido tamaño efectivo poblacional. Sin embargo, no puede descartarse que parte de las diferencias observadas sean consecuentes a un efecto de muestreo.

\begin{tabular}{lcccccc}
\hline País/Región & $\mathbf{n}$ & $\mathbf{q}$ & $\mathbf{( + / + )}$ & $(+/-)$ & $(-l-)$ & Referencia \\
\hline Alemania & 578 & 0,55 & 0,24 & 0,43 & 0,33 & Geyer et. al, 2005 \\
EE.UU. & 161 & 0,51 & 0,26 & 0,46 & 0,28 & Neff et. al, 2004 \\
Reino Unido & 94 & 0,60 & 0,15 & 0,51 & 0,34 & \\
Australia & 33 & 0,56 & 0,12 & 0,64 & 0,24 & Mealey et. al, 2005 \\
Francia & 25 & 0,64 & 0,20 & 0,32 & 0,48 & Hugnet et. al, 2004 \\
Japón & 12 & 0,58 & 0,25 & 0,33 & 0,42 & Kawabata et. al, 2005 \\
Europa & 1310 & 0,48 & 0,28 & 0,47 & 0,25 & Firdova et. al, 2016 \\
Argentina & 30 & 0,32 & 0,53 & 0,30 & 0,17 & Este trabajo \\
\hline
\end{tabular}

Tabla 4. Cuadro comparativo de la frecuencia de la mutación (q) nt23o [del4] del gen $A B C B 1$ canino entre las frecuencias genotípicas informadas en otros países y las encontradas en este estudio para la raza collie. (+): alelo normal; (-): alelo mutado; (n): número de animales; (+/+): genotipo homocigota normal; (+/-): genotipo heterocigota; (-/-): genotipo homocigota mutado.

En la estructura poblacional de los animales utilizados en este trabajo, se puede observar a una población en desequilibrio y marcadas diferencias genéticas entre la raza collie y los otros grupos analizados. Este fenómeno es común en la especie canina teniendo en cuenta los métodos utilizados en la formación de las distintas razas (Calboli et al., 2008). 


\section{Conclusiones}

Un método rápido y específico para determinar el genotipo de los perros con la deleción nt230 [del4] del gen $A B C B 1$ es de gran utilidad. Estos pacientes muestran intolerancia a drogas utilizadas de forma rutinaria en medicina veterinaria como la ivermectina, la vincristina y la doxorrubicina, provocando la acumulación de la droga en el cerebro $y$, en consecuencia, neurotoxicidad severa.

La detección por pirosecuenciación de esta mutación servirá tanto para guiar los tratamientos que utilizan drogas sustrato de la Pgp, como para eliminar esta deleción en los criaderos de las razas de pastores y sus cruzas.

Además, este trabajo aporta una primera aproximación a la prevalencia de la mutación en la provincia de Buenos Aires.

\section{Agradecimientos}

Este estudio fue financiado por el Consejo Nacional de Investigaciones Científicas y Técnicas (CONICET). Los autores agradecen a la Méd. Vet. Lorena Petetta por su colaboración con el muestreo y a la Trad. L. Adriana Di Maggio por la corrección y edición del manuscrito.

\section{Conflicto de intereses}

Todos los autores declaran que no existe conflicto de intereses, incluyendo las relaciones financieras, personales o de otro tipo que pudieran influir en el trabajo.

\section{Bibliografía}

Baars C, Leeb T, von Klopmann T, Tipold A, Potschka H. 2008. Allele-specific polymerase chain reaction diagnostic test for the functional MDR1 polymorphism in dog. The Veterinary Journal. 177:394-7.

doi: 10.1016/j.tvjl.2007.05.020

Calboli FC, Sampson J, Fretwell N, Balding DJ. 2008. Population structure and inbreeding from pedigree analysis of purebred dogs. Genetics. 179(1):593-601.

doi: 10.1534/genetics.107.084954

Fromm MF. 2003. Importance of P-glycoprotein for drug disposition in humans. European Journal of Clinical Investigation. 33(2):6-9.

Gramer I, Leidolf R, Döring B, Klintzsch S, Krämer EM, Yalcin E, Petzinger E, Geyer J. 2011. Breed distribution of the nt23o(del4) MDR1 mutation in dogs. The Veterinary Journal. 189(1):67-71.

doi: 10.1016/j.tvjl.2010.06.012

Geyer J, Döring B, Godoy JR, Leidolf R, Moritz A, Petzinger E. 2005. Frequency of the nt230 (del4) MDR1 mutation in Collies and related dog breeds in Germany. Journal of Veterinary Pharmacology and Therapeutics. 28:545-51.

doi: 10.1111/j.1365-2885.2005.00692.x

Huebner J, Kühnlein P, Langbein-Detsch I, Müller E. 2007. LABOKLIN. Canine MDR1 mutation breed disposition and prevalence in dogs in Germany. [ONLINE] Disponible en: http:// v17.laboklin.com/. Consultado 10/10/2017.

Hugnet C, Bentjen SA, Mealey KL. 2004. Frequency of the mutant MDR1 allele associated with multidrug sensitivity in a sample of collies from France. Journal of Veterinary Pharmacology and Therapeutics. 27:227-9.

doi: 10.1111/j.1365-2885.2004.00585.x

Firdova Z, Turnova E, Bielikova M, Turna J, Dudas A. 2016. The prevalence of ABCB1:C. 227_23odelATAG mutation in affected dog breeds from European countries. Research in Veterinary Science. 106:89-92.

doi: 10.1016/j.rvsc.2016.03.016

Kawabata A, Momoi Y, Inoue-Murayama M, Iwasaki T. 2005. Canine mdr1 gene mutation in Japan. Journal of Veterinary Medical Science. 67:1103-07.

doi: 10.1292/jvms.67.1103

Löscher W, Potschka H. 2005. Role of drug efflux transporters in the brain for drug disposition and treatment of brain diseases. Progress in Neurobiology. 76:22-76.

doi: 10.1016/j.pneurobio.2005.04.006

Mealey KL, Bentjen SA, Gay JM, Cantor GH. 2001. Ivermectin sensitivity in Collies is associated with a deletion mutation of the mdr1 gene. Pharmacogenetics. 11:727-33.

doi: 10.1097/00008571-200111000-00012

Mealey KL, Munyard KA, Bentjen SA. 2005. Frequency of the mutant mdr1 allele associated with multidrug sensitivity in a sample of herding breed dogs living in Australia. Veterinary Parasitology. 131:193-6.

doi: 10.1016/j.vetpar.2005.05.004

Neff MW, Robertson KR, Wong AK, Safra N, Broman KW, Slatkin M, Mealey KL, Pedersen NC. 2004. Breed distribution and history of canine mdr1-1, a pharmacogenetic mutation that marks the emergence of breeds from the collie lineage. Proceedings of the National Academy of Science of the United States of America. 101(32):11725-30. doi: 10.1073/pnas.0402374101 
Nelson OL, Carsten E, Bentjen SA, Mealey KL. 2003. Ivermectin toxicity in an Australian Shepherd dog with the MDR1 mutation associated with ivermectin sensitivity in Collies. Journal of Veterinary Internal Medicine. 17:354-6.

doi:10.1111/j.1939-1676.2003.tbo2461.x

Roulet A, Puel O, Gesta S, Lepage JF, Drag M, Soll M, Alvinerie M, Pineau T. 2003. MDR1-deficient genotype in Collie dogs hypersensitive to the Pglycoprotein substrate ivermectin. European Journal of Pharmacology. 460:85-91.

doi: 10.1016/Soo14-2999(02)02955-2
Schinkel AH. 1999. P-glycoprotein, a gatekeeper in the blood-brain barrier. Advanced Drug Delivery Reviews. 36:179-94.

doi: 10.1016/So169-409X(98)ooo85-4

Stiedl C, Weber K. 2017. Fast and simple detection methods for the 4-base pair deletion of canine MDR1/ABCB1 gene by PCR and isothermal amplification. Journal of Veterinary Diagnostic Investigation. 29(2):176-80.

doi: $10.1177 / 1040638716683213$ 


\section{Material complementario}

\section{Genotipificación por secuenciación directa}

Los cebadores para la PCR fueron diseñados utilizando el programa Annhyb (http://bioinformatics.org/annhyb/) y la secuencia de ADN reportada en GenBank (http://www.ncbi. nlm.nih.gov/genbank/) (Tabla 1).

\section{Reacción de PCR}

- 20 ng de ADN

- Buffer de reacción 1X (50 mM KCl, 11 mM Tris, $\mathrm{pH}$ = 8,30)

- $\mathrm{MgCl} 20,5 \mathrm{mM}$

- Potenciador (enhancer) o,o8X

- dNTP $100 \mu \mathrm{M}$ de cada uno

- Cebadores o,2 $\mu \mathrm{M}$ cada uno

- polimerasa Taq 0,75 U (Kit T-Holmes ADN polimerasa, INBIO Highway, Tandil, Argentina).

\section{Condiciones de ciclado}

- Desnaturalización inicial de 5 min a $94{ }^{\circ} \mathrm{C}$

$$
\begin{array}{ll}
-35 \text { ciclos de } & -30 \mathrm{~s} \text { a } 94^{\circ} \mathrm{C} \\
& -45 \mathrm{~s} \text { a } 58^{\circ} \mathrm{C} \\
& -45 \mathrm{~s} \text { a } 72^{\circ} \mathrm{C}
\end{array}
$$

- Elongación final de 10 min a $72{ }^{\circ} \mathrm{C}$

Los productos de PCR fueron purificados con polietilenglicol (PEG) 8000 y secuenciados en un secuenciador automático Genetic Analyzer 3500 (Applied Biosystems®) utilizando el kit comercial $\left(\right.$ BigDye $^{\mathrm{TM}}$ Terminator v3.1 Cycle Sequencing Kit - Applied Biosystem by Thermo Fisher Scientific).

Las secuencias fueron alineadas y analizadas utilizando los programas para análisis de secuencias DNAMAN 4.15, Lynnon BioSoft, Vaudreuil, Quebec, Canadá y Chromas 2.4, Technelysium, South Brisbane, Australia.

\section{Genotipificación por pirosecuenciación}

Se llevó a cabo en dos etapas.

1) Condiciones de ciclado utilizando los cebadores específicos (F-mdr1 y R-mdr1-M13):

- Desnaturalización inicial de $3 \mathrm{~min}$ a $94{ }^{\circ} \mathrm{C}$

- 15 ciclos de $\quad-30 \mathrm{~s} \mathrm{a} 94^{\circ} \mathrm{C}$

$-45 \mathrm{~s}$ a $58^{\circ} \mathrm{C}$

$-45 \mathrm{~s}$ a $72{ }^{\circ} \mathrm{C}$

2) Incorporación de un cebador biotinilado M13-Biot con temperatura de hibridación de $52{ }^{\circ} \mathrm{C}$.

\section{Condiciones del ciclado}

- 30 ciclos de $\quad-30$ s a $94^{\circ} \mathrm{C}$

$-45 \mathrm{~s}$ a $52^{\circ} \mathrm{C}$

$-45 \mathrm{~s}$ a $72^{\circ} \mathrm{C}$

- Elongación final de $72{ }^{\circ} \mathrm{C} 10 \mathrm{~min}$.

Los productos de PCR fueron purificados captando los fragmentos biotinilados con perlas de sefarosa cubiertas con estreptavidina (Streptavidin Sepharose ${ }^{\mathrm{TM}}$ High Performance, GE Healthcare). Los fragmentos biotinilados se inmovilizaron y utilizaron como molde para realizar la reacción de pirosecuenciación, incorporando el cebador interno INT-mdr1 (o,5 $\mu \mathrm{M})$ usando el kit comercial (Pyro Gold Reagent Kit, Biotage, Uppsala, Suecia) y PyroMark Prep Workstation (QIAGEN, Hilden, Alemania). Las muestras se tipificaron en un pirosecuenciador PSQTM96 System instrument (QIAGEN) y los resultados se analizaron con el programa PSQ HS96A (QIAGEN). 